\title{
STABLE RANGE ONE FOR RINGS WITH MANY IDEMPOTENTS
}

\author{
VICTOR P. CAMILLO AND HUA-PING YU
}

\begin{abstract}
An associative ring $R$ is said to have stable range 1 if for any $a, b \in R$ satisfying $a R+b R=R$, there exists $y \in R$ such that $a+b y$ is a unit. The purpose of this note is to prove the following facts. Theorem 3: An exchange ring $R$ has stable range 1 if and only if every regular element of $R$ is unit-regular. Theorem 5: If $R$ is a strongly $\pi$-regular ring with the property that all powers of every regular element are regular, then $R$ has stable range 1 . The latter generalizes a recent result of Goodearl and Menal [5].
\end{abstract}

Let $R$ be an associative ring with identity. $R$ is said to have stable range 1 if for any $a, b \in R$ satisfying $a R+b R=R$, there exists $y \in R$ such that $a+b y$ is a unit. This definition is left-right symmetric by Vaserstein [9, Theorem 2]. Furthermore, by a theorem of Kaplansky, all one-sided units are two-sided in rings having stable range 1 (cf. Vaserstein [10, Theorem 2.6]). It is well known that a (von Neumann) regular ring $R$ has stable range 1 if and only if $R$ is unit-regular (see, for example, Goodearl [4, Proposition 4.12]).

Call a ring $R$ strongly $\pi$-regular if for every element $a \in R$ there exist a number $n$ (depending on $a$ ) and an element $x \in R$ such that $a^{n}=a^{n+1} x$. This is in fact a two-sided condition [3]. It is an open question whether all strongly $\pi$-regular rings have stable range 1 . Goodearl and Menal [5] proved that strongly $\pi$-regular rings are unit-regular and, hence, have stable range 1 (Theorem 5.8, p. 278).

In this note we first extend the above result for von Neumann regular rings to a larger class of rings, which includes all strongly $\pi$-regular rings, $\pi$-regular rings, von Neumann regular rings, and algebraic algebras. As an application of this, we prove that a strongly $\pi$-regular ring $R$ has stable range 1 if powers of every regular element are regular. The latter is a generalization of the abovementioned result of Goodearl and Menal for strongly $\pi$-regular regular rings. As one can see from our proofs, rings in these classes have a large supply of idempotents.

Throughout, $R$ stands for an associative ring with identity and $J(R)$ for the Jacobson radical of $R$. Modules are unitary right $R$-modules except otherwise specified. For other undefined terms, readers are referred to [4].

Let $M_{R}$ be a right $R$-module. Following Crawley and Jonsson [2], $M_{R}$ is said to have the exchange property if for every module $A_{R}$ and any two

Received by the editors January 24, 1994 and, in revised form, May 3, 1994; originally communicated to the Proceedings of the AMS by K. A. Goodearl.

1991 Mathematics Subject Classification. Primary 16D70, 16P70.

Key words and phrases. Stable range one, exchange ring, strongly $\pi$-regular ring. 
decompositions of $A_{R}$

$$
A_{R}=M^{\prime} \oplus N=\bigoplus_{i \in I} A_{i}
$$

where $M_{R}^{\prime} \cong M_{R}$, there exist submodules $A_{i}^{\prime} \subseteq A_{i}$ such that

$$
A=M^{\prime} \oplus\left(\bigoplus_{i \in I} A_{i}^{\prime}\right)
$$

$M_{R}$ is said to have the finite exchange property if the above condition is satisfied whenever the index set $I$ is finite. Many familiar classes of modules have the exchange property or the finite exchange property, see Zimmermann-Huisgen and Zimmermann [12] for a list of these classes of modules.

Warfield [8] introduced the class of exchange rings. He called a ring $R$ an exchange ring if $R_{R}$ has the exchange property above and proved that this definition is left-right symmetric. The class of exchange rings is quite large. Call a ring $R$ semiregular (semi- $\pi$-regular, semi-strongly $\pi$-regular) if $R / J(R)$ is regular ( $\pi$-regular, strongly $\pi$-regular) and idempotents can be lifted modulo $J(R)$. It is easy to verify that the following classes of rings (in the order of containments) are all contained in the class of exchange rings: (1) local rings; (2) semiperfect rings; (3) semiregular rings; (4) semistrongly $\pi$-regular rings; (5) semi- $\pi$-regular rings (see, for example, Stock [7, p. 440]).

The following characterizations of the finite exchange property for projective modules were given by Nicholson [6, Proposition 2.9].

Lemma 1 (Nicholson). The following conditions are equivalent for a projective module $P$ :

(1) $P$ has the finite exchange property.

(2) If $P=M_{1}+M_{2}+\cdots+M_{n}$ where $M_{i}$ are submodules, there is a decomposition $P=P_{1} \oplus P_{2} \oplus \cdots \oplus P_{n}$ with $P_{i} \subseteq M_{i}$ for each $i$.

(3) If $P=M+N$ where $M$ and $N$ are submodules, there exists a summand $P_{1}$ of $P$ such that $P_{1} \subseteq M$ and $P=P_{1}+N$.

The original definition of stable range 1 for an arbitrary ring $R$ is equivalent to the condition that for any $a, x, b \in R$ satisfying $a x+b=1$, there exists $y \in R$ such that $a+b y$ is a unit in $R$. The next lemma says that, for exchange rings, the element $b$ in the latter condition can be further restricted to idempotents.

Lemma 2. Let $R$ be an exchange ring, then the following conditions are equivalent:

(1) $R$ has stable range 1 .

(2) For any $a \in R, e^{2}=e \in R$, if $a x+e=1$ for some $x \in R$, then there exists $y \in R$ such that $a+e y=u$ is a unit.

Proof. (1) $\Rightarrow(2)$ : Trivial. (2) $\Rightarrow(1)$ : Assume that $a R+b R=R . R$ is exchange, there exists an idempotent $e^{2}=e \in b R$ such that $(1-e) R \oplus e R=R$ where $(1-e) R \subseteq a R$ and $e R \subseteq b R$, by Lemma 1. So we have $a x+e=1$ for some $x \in R$. By assumption, there exists $y \in R$ such that $a+e y=u$ is a unit; hence, $a+b r y=u$ is a unit where $e=b r$.

Recall that for a regular ring $R, R$ has stable range 1 if and only if $R$ is unit-regular. We now extend this to exchange rings. 
Theorem 3. An exchange rings $R$ has stable range 1 if and only if every regular element of $R$ is unit-regular in $R$.

Proof. $\Rightarrow$ : Let $a x a=a$; then $a x+(1-a x)=1$. By the assumption on $R$, there exists $y \in R$ such that $a+(1-a x) y=u$ is a unit in $R$. Multiplying both sides of the latter equality by $a x$ on the left, we have that axa $=a=$ $a x u, a u^{-1}=a x$, so $a u^{-1} a=a$, and $a$ is unit-regular.

$\Leftarrow$ : By Lemma 2, we need only show that if $a x+e=1$ with $e^{2}=e$, there exists an element $y \in R$ such that $a+e y$ is a unit.

We first show that, without loss of generality, we may assume $a x a=a$. In fact, if $a x a \neq a$, put $f=a x$ and $r=f a-a$; then $r x=0$. Letting $a^{\prime}=a+r$, we have $a^{\prime} x=a x+r x=a x+0=a x=f, a^{\prime} x a^{\prime}=f a^{\prime}=f a+f r=f a+0=$ $a+r=a^{\prime}$. To see that $f r=0$, notice that $f=a x=1-e$ is an idempotent. Now if $a^{\prime}+e y$ is a unit for some $y \in R$ and $f r=0$ implies $r \in(1-f) R=e R$, we have

$$
a^{\prime}+e y=a+r+e y=a+e s+e y=a+e(s+y)
$$

is a unit.

So we can assume that $a x+e=1$, where $e^{2}=e$ and $a x a=a$. Notice that $a x a=a$ if and only if $e a=0$. Since we assume that every regular element is unit-regular, there exists a unit $u \in R$ such that $a u a=a$. Then we have $1-e=a x=(a u a) x=(a u)(a x)=a u(1-e)$.

$$
\begin{aligned}
(a u-e)^{2} & =(a u-e)(a u-e)=a u a u-a u e-e a u+e \\
& =a u-a u e-0+e=a u(1-e)+e=1 .
\end{aligned}
$$

So $a u-e=v$ is a unit, therefore $a-e u^{-1}=v u^{-1}$ is a unit.

For some other equivalent characterizations of stable range 1 for exchange rings, see $\mathrm{Yu}$ [11, Theorem 9].

While the question of whether all strongly $\pi$-regular rings have stable range 1 remains open, we now can reduce this to a unit-regularity problem.

Corollary 4. A strongly $\pi$-regular ring $R$ has stable range 1 if and only if every regular element of $R$ is unit-regular in $R$.

Corollary 4 should be compared with an analogous result of Goodearl and Menal [5, Theorem 6.1], which says that a strongly $\pi$-regular ring $R$ has stable range 1 if and only if every nilpotent regular element of each corner of $R$ is unit-regular in that corner. By a corner of a ring $R$, they mean any (nonunital) subring $e R e$ where $e$ is an idempotent in $R$. While it is true that an element $x \in e R e$ is regular in $e R e$ if and only if it is regular in $R$, the same is not true for unit-regularity.

One of the known cases where strongly $\pi$-regular rings have stable range 1 was Theorem 5.8 of Goodearl and Menal [5]: a strongly $\pi$-regular regular ring has stable range 1. As an application of our Theorem 3, we now extend this to the following:

Theorem 5. Let $R$ be a strongly $\pi$-regular ring. If all powers of every regular element are regular, then $R$ has stable range 1 .

The proof we are going to give is a modification of Goodearl and Menal's proof in [5]. In order to make our paper self-contained, we present here a complete proof, although a portion of it is just a verbatim adoption of their argument 
in von Neumann regular context. Also, we need the following lemma, which is a combination of Azumaya's Theorem 3 in [1] and Dischinger's Theorem 1 in [3].

Lemma 6 (Azumaya, Dischinger). For every element $x \in R$ of a strongly $\pi$ regular ring $R$, there exist $a \in R$ and an integer $n \geq 1$ such that $x a=a x$ and $x^{n}=a x^{n+1}=x^{n+1} a$.

Proof of Theorem 5. Let $x \in R$ be a regular element with $x y x=x$. It suffices to prove, by Corollary 4 , that $x$ is unit-regular.

Set $K_{i}=\operatorname{rann}\left(x^{i}\right)$ for all $i=0,1,2, \ldots$.

Claim 1. There exists an integer $n \geq 1$ such that $x R+K_{n}=R$ and $x^{n} R \cap K_{1}=$ 0 .

In fact, by Lemma 6 , there exist an integer $n \geq 1$ and $a \in R$ such that $a x=x a$ and $x^{n}=a x^{n+1}=x^{n+1} a$. Pick any $r \in R$, we have $x^{n} r=x^{n+1} a r$ and $x^{n}(r-x a r)=0$; thus $r-x a r \in K_{n}$ and $r \in x R+K_{n}$. Therefore, $x R+K_{n}=R$.

Since $x^{n}=a x^{n+1}$, it is clear that $K_{n}=K_{n+1}$. If $x^{n} d \in x^{n} R \cap K_{1}$, then $x x^{n} d=0$ and $d \in K_{n+1}=K_{n}$, so $x^{n} d=0$, i.e., $x^{n} R \cap K_{1}=0$.

Claim 2. $x R+K_{i}$ are direct summands of $R_{R}$ for all $i \geq 1$.

Since $x^{i}$ is regular for all $i \geq 2$ by our assumption on $R$, we may assume $x^{i} y_{i} x^{i}=x^{i}$ for some $y_{i} \in R$ for $i \geq 2$. Then $K_{i}=\left(1-y_{i} x^{i}\right) R$. It is easy to check that

$$
x R+\left(1-y_{i} x^{i}\right) R=y_{i} x^{i} x R+\left(1-y_{i} x^{i}\right) R .
$$

We check below that the element $y_{i} x^{i} x$ is actually von Neumann regular:

$$
y_{i} x^{i} x \cdot y_{i+1} x^{i} \cdot y_{i} x^{i} x=y_{i} x^{i} x y_{i+1} x^{i} x=y_{i} x^{i} x \text {. }
$$

Put $e_{i}=y_{i} x^{i} x y_{i+1} x^{i}$ and $f_{i}=1-y_{i} x^{i}$, then $e_{i} f_{i}=f_{i} e_{i}=0$. We see that $e_{i}$ and $f_{i}$ are orthogonal idempotents, hence $e_{i}+f_{i}$ is an idempotent. But $y_{i} x^{i} x R=e_{i} R$, so $x R+K_{i}=e_{i} R+f_{i} R=\left(e_{i}+f_{i}\right) R$ is a direct summand of $R_{R}$.

Recall that we assume $x y x=x$, so $x R+K_{1}$ is a direct summand of $R_{R}$ for the same reason.

Claim 3. $x^{i} R \cap K_{1}$ are all direct summands of $R_{R}$ for all $i \geq 1$.

First, we show $x^{i} R \cap K_{1}=x^{i} K_{i+1}$. Since $x^{i} K_{i+1} \subset x^{i} R$ and $x^{i} K_{i+1} \subset K_{1}$, $x^{i} K_{i+1} \subset x^{i} R \cap K_{1}$; on the other hand, pick any $x^{i} r \in x^{i} R \cap K_{1}, x x^{i} r=x^{i+1} r=$ $0, r \in K_{i+1}$, so $x^{i} r \in x^{i} K_{i+1}, x^{i} R \cap K_{1} \subset x^{i} K_{i+1}$.

Second, recall that we assume $x^{i} y_{i} x^{i}=x^{i}$, so that $K_{i+1}=\left(1-y_{i+1} x^{i+1}\right) R$, and we see that $x^{i} R \cap K_{1}=x^{i} K_{i+1}=x^{i}\left(1-y_{i+1} x^{i+1}\right) R$. We check below that $x^{i}\left(1-y_{i+1} x^{i+1}\right)$ is von Neumann regular:

$$
\begin{aligned}
x^{i}(1 & \left.-y_{i+1} x^{i+1}\right) \cdot y_{i} \cdot x^{i}\left(1-y_{i+1} x^{i+1}\right)=\left(x^{i}-x^{i} y_{i+1} x^{i+1}\right) y_{i} x^{i}\left(1-y_{i+1} x^{i+1}\right) \\
& =\left(1-x^{i} y_{i+1} x\right) x^{i} y_{i} x^{i}\left(1-y_{i+1} x^{i+1}\right)=\left(1-x^{i} y_{i+1} x\right) x^{i}\left(1-y_{i+1} x^{i+1}\right) \\
& =\left(x^{i}-x^{i} y_{i+1} x^{i+1}\right)\left(1-y_{i+1} x^{i+1}\right)=x^{i}\left(1-y_{i+1} x^{i+1}\right)\left(1-y_{i+1} x^{i+1}\right) \\
& =x^{i}\left(1-y_{i+1} x^{i+1}\right) .
\end{aligned}
$$

Therefore $x^{i} R \cap K_{1}=x^{i} K_{i+1}$ is a direct summand of $R_{R}$.

Inasmuch as $x y x=x, x R \cap K_{1}=x K_{2}$ is a direct summand of $R_{R}$. 
Claim 4. $\left(x R+K_{m}\right) / x R \cong K_{1} / x^{m} R \cap K_{1}$ for all $m$.

Every right ideal involved here is a direct summand of $R_{R}$ by Claims 2 and 3. We have the ascending and descending chains of direct summands

$$
\begin{gathered}
x R \subset^{\oplus} x R+K_{1} \subset^{\oplus} x R+K_{2} \subset^{\oplus} \ldots \subset^{\oplus} x R+K_{m}, \\
K_{1}^{\oplus} \supset x R \cap K_{1}^{\oplus} \supset x^{2} R \cap K_{1}^{\oplus} \supset \ldots \oplus \supset x^{m} R \cap K_{1}
\end{gathered}
$$

which give us the decompositions

$$
\begin{gathered}
\left(x R+K_{m}\right) / x R \cong \bigoplus_{i=0}^{m-1}\left(x R+K_{i+1}\right) /\left(x R+K_{i}\right), \\
K_{1} /\left(x^{m} R \cap K_{1}\right) \cong \bigoplus_{i=0}^{m-1}\left(x^{i} R \cap K_{1}\right) /\left(x^{i+1} R \cap K_{1}\right) .
\end{gathered}
$$

So if we can show that

$$
\left(x R+K_{i+1}\right) /\left(x R+K_{i}\right) \cong\left(x^{i} R \cap K_{1}\right) /\left(x^{i+1} R \cap K_{1}\right)
$$

for all $i$, we are done.

First we note that

$$
\begin{aligned}
\left(x R+K_{i+1}\right) /\left(x R+K_{i}\right) & =\left(x R+K_{i}+K_{i+1}\right) /\left(x R+K_{i}\right) \\
\cong K_{i+1} /\left[\left(x R+K_{i}\right)\right. & \left.\cap K_{i+1}\right]=K_{i+1} /\left[\left(x R \cap K_{i+1}\right)+K_{i}\right] .
\end{aligned}
$$

As $x^{i} K_{i+1} \subseteq x^{i} R \cap K_{1}$ and $x^{i}\left[\left(x R \cap K_{i+1}\right)+K_{i}\right] \subseteq x^{i+1} R \cap K_{1}$, left multiplication by $x^{i}$ gives a module homomorphism

$$
f: K_{i+1} /\left[\left(x R \cap K_{i+1}\right)+K_{i}\right] \rightarrow\left(x^{i} R \cap K_{1}\right) /\left(x^{i+1} R \cap K_{1}\right) .
$$

The map $f$ is epic: Pick any $r \in x^{i} R \cap K_{1}, r=x^{i} a$ for some $a \in R$. But $x^{i+1} a=x r=0$; then $a \in K_{i+1}$. So $f(a)=r$.

The map $f$ is monic: Suppose $z \in K_{i+1}$ and $x^{i} z \in x^{i+1} R \cap K_{1}$; then we have $x^{i} z=x^{i+1} b$ for some $b \in R$ and $x^{i+1} b=x\left(x^{i} z\right)=0$, whence $x b \in K_{i+1} \cap x R$. Since $x^{i}(z-x b)=0, z-x b \in K_{i}$; thus $z \in\left(x R \cap K_{i+1}\right)+K_{i}$, i.e. $f$ is monic.

We have proved that $f$ is an isomorphism.

Claim 5. $x$ is unit-regular, i.e., there exists a unit $u \in R$ such that $x u x=x$.

It follows from Claims 1 and 4 that

$$
\left(x R+K_{n}\right) / x R=R / x R \cong K_{1} /\left(x^{n} R \cap K_{1}\right)=K_{1} / 0=K_{1} .
$$

It is assumed that $x y x=x$; hence

$$
R=y x R \oplus K_{1}=x R \oplus(1-x y) R .
$$

So $K_{1} \cong(1-x y) R$. Denote this isomorphism by $\alpha$. Also, the restriction of the left multiplication by $x$ gives an isomorphism $\beta$ from $y x R$ to $x R$. Define $u \in \operatorname{end}\left(R_{R}\right)=R$ to be the direct sum of $\alpha$ and $\beta^{-1}$; it is easy to check that $u$ is a unit in $R$ and $x u x=x$.

The above proof actually proves the following more general statement: 
Theorem 7. For an exchange ring $R$, if powers of every von Neumann regular element are von Neumann regular and for every von Neumann regular element $x \in R$ there exists an integer $n \geq 1$ such that $x^{n} R=x^{n+1} R$ and $R x^{n}=R x^{n+1}$, then $R$ has stable range one.

Proof. $x^{n} R=x^{n+1} R$ implies $x R+K_{n}=R$ and $R x^{n}=R x^{n+1}$ implies $K_{n}=$ $K_{n+1}$; hence $x^{n} R \cap K_{1}=0$. So Claim 1 is valid. Claims 2 and 3 use only the property that powers of every regular element are regular and so are still valid in this case. Claims 4 and 5 have nothing to do with the strongly $\pi$-regularity of $R$ and therefore are also valid here. Finally, the conclusion follows from Theorem 3.

We conclude this note by giving two examples. One shows that our generalization of Goodearl and Menal's result on strongly $\pi$-regular regular rings to Theorem 5 is nontrivial, the other shows that the converse of Theorem 5 is false.

Example 8. Let $F$ be any field, $R=\left(\begin{array}{cc}F & F \\ 0 & F\end{array}\right)$.

$R$ is obviously Artinian and hence strongly $\pi$-regular. One checks that an element $x \in R$ is von Neumann regular in $R$ if and only if $x$ is not nilpotent. So powers of every regular element in $R$ are regular. But $J(R)=\left(\begin{array}{ll}0 & F \\ 0 & 0\end{array}\right) \neq 0$, therefore $R$ is not regular. This shows that our generalization of Goodearl and Menal's result on regular strongly $\pi$-regular rings to Theorem 5 is nontrivial.

Example 9. Let $R$ be the $2 \times 2$ matrix ring over $F[x] /\left(x^{2}\right)$, where $F$ is a field.

Clearly, $R$ is a finite-dimensional algebra and hence strongly $\pi$-regular. Of course, $R$ has stable range 1 . But not all the powers of every regular element are regular in $R$. Take $a=\left(\begin{array}{ll}0 & 1 \\ 0 & x\end{array}\right)$ and $u=\left(\begin{array}{ll}0 & 1 \\ 1 & 0\end{array}\right)$; it is easy to see that $a u a=a$. But $a^{2}=\left(\begin{array}{ll}0 & x \\ 0 & 0\end{array}\right)$ is not regular. So the condition that powers of every regular element are regular is sufficient but not necessary for strongly $\pi$-regular rings to have stable range 1 .

\section{ACKNOWLEDGMENT}

Example 9 was communicated to the authors by Professor K. R. Goodearl. We thank him for this example.

\section{REFERENCES}

1. G. Azumaya, Strongly $\pi$-regular rings, J. Fac. Sci. Hokkaido Univ. 13 (1954), 34-39.

2. P. Crawley and B. Jonsson, Refinements for infinite direct decompositions of algebraic systems, Pacific J. Math. 14 (1964), 797-855.

3. F. Dischinger, Sur les anneaux fortement $\pi$-reguliers, C. R. Acad. Sci. Paris Ser. A 283 (1976), 571-573.

4. K. R. Goodearl, Von Neumann regular rings, Pitman, London, 1979.

5. K. R. Goodearl and P. Menal, Stable range one for rings with many units, J. Pure Appl. Algebra 54 (1988), 261-287.

6. W. K. Nicholson, Lifting idempotents and exchange rings, Trans. Amer. Math. Soc. 229 (1977), 269-278.

7. J. Stock, On rings whose projective modules have the exchange property, J. Algebra 103 (1986), 437-453. 
8. R. B. Warfield, Exchange rings and decomposition of modules, Math. Ann. 199 (1972), 31-36.

9. L. N. Vaserstein, Stable rank of rings and dimensionality of topological spaces, Functional Anal. Appl. 5 (1971), 102-110.

10. __ Bass's first stable range condition, J. Pure Appl. Algebra 34 (1984), 319-330.

11. H. P. Yu, Stable range one for exchange rings, J. Pure Appl. Algebra (to appear).

12. B. Zimmermann-Huisgen and $\mathrm{W}$. Zimmermann, Classes of modules with the exchange property, J. Algebra 88 (1984), 416-434.

Department of Mathematics, The University of Iowa, Iowa City, Iowa 52242

E-mail address: vcamillo@math.uiowa.edu

hpyu@math. uiowa.edu 